\title{
Study of Top and Bottom Photonic Gratings on GaN LED With Error Grating Models
}

\author{
Simeon S. Trieu, and Xiaomin Jin
}

\begin{abstract}
The gallium nitride (GaN) light-emitting-diode (LED) top-bottom (or transmission-reflection) grating simulation results with error grating model are presented. The microstructure GaN bottom hole and top pillar gratings are calculated and compared with the non-grating (flat) case. Grating shapes simulated are either conical or cylindrical. A direct comparison of 181 different combined transmission-reflection grating cases using the finite difference time domain method is presented. The simulation results show that simple or direct combinations of the optimized top grating with the optimized bottom grating only produce a $42 \%$ light extraction improvement compared to the non-grating case, which is much lower than that of an optimized single grating case. This is due to the mismatch of grating parameters with the direct addition of the second grating structure, which changes the optical field distribution in the LEDs. Therefore, it is very important to optimize both top and bottom gratings simultaneously for the double-grating design. We also show the optimization of a double grating structure can achieve better performance than a single grating. Finally, transmission-reflection error gratings are also presented. It is also the first time to present randomization in GaN LED grating design and its effects in fabrication. Our data shows that the favorable light extraction improvement is at approximately $10-15 \%$ randomization. The randomization can achieve $230 \%$ improvement over the original grating at a randomization intensity factor of $\mathbf{1 2 . 8 \%}$.
\end{abstract}

Index Terms-Error grating, finite-difference time domain, light emitting diode, photonic crystal.

\section{INTRODUCTION}

$\mathbf{L}$ IGHT EMITTING diodes (LEDs) have become an area of intensive research in an effort to develop more efficient, brighter, and greener light sources. Light emitting diodes are used extensively in displays, light bulbs, photonics, and machine vision. To meet the needs of these applications, light sources must be low-power, generate less heat, and achieve high luminosity. There are two methods to improve these factors in LEDs: 1) increase electroluminescent efficiency; and 2) increase light extraction efficiency [1]. Therefore, it is crucial to improve light extraction efficiency of gallium nitride (GaN) LEDs to reduce energy consumption, limit heat generation, and increase luminosity. Usually GaN LEDs have poor light extraction efficiencies due to: 1) the high refractive index of semiconductor based devices that results in a low critical angle and total internal reflection; 2) the absorption of light within the device due to dislocations and defects within the GaN crystal; and 3) inefficient device design and structure [2]-[4].

One of the key limitations for light emissive devices is the light trapping due to the device layers' low critical angle. The light generated within the device is totally internally reflected. A number of solutions have been proposed to solve this problem. A resin can be affixed over the device to create a larger escape angle to air [5]. To further mitigate the problems of total internal reflection, the emission surface can be patterned to form a transmission grating that offers the trapped light more angles of escape. This can be done with a variety of methods including wet etching with an amorphous sacrificial layer [6]-[9] or by laser etching to obtain a more periodic spacing [10]-[14]. In addition, it has been shown that the same patterning can also apply to a Ag reflector plate in either pillar or hole grating shapes to form a reflection grating [15], [16].

The second inefficiency of the device is due to absorption losses due to dislocation and defects within the GaN crystal. This is a critical aspect of light extraction efficiency since the photon is not escaping from the device, but also because photon absorption within the semiconductor generates heat. So, it is important to extract light from the device both in greater quantity and speed before recombination [4], [17]. A grating structure will help solve this issue by creating more angles of escape. Grating structures can be patterned with many shapes including: pyramidal, spherical, conical, cylindrical, and so on, but only a few can be fabricated with great success. For example, with the modified laser lift off (M-LLO) technique, holes can be patterned at a $4 \mu \mathrm{m}$ period instead of etching a random structure. The technique uses a sapphire backplane, UV light, and a high power $\mathrm{KrF}$ laser to etch the nanostructure onto u-GaN. In the experiment at Peking University (PKU), grating depths are varied from $75 \mathrm{~nm}$ to $120 \mathrm{~nm}$ [18]. Other fabrication techniques exist, such as imprint lithography, that can produce similar air holes that measure $180 \mathrm{~nm}$ in diameter, with a depth of $67 \mathrm{~nm}$, and a period of $295 \mathrm{~nm}$ [20].

Etching a structure on the extraction surface and/or on the bottom reflection surface commonly solves the light trapping issue. The single grating simulation has been studied 
intensively already. However, there is no publication directly comparing the effect of top grating, bottom grating, and combinations of both in theory. It is also not practical to fabricate all the double grating cases to obtain the best cases. Even our calculation here is only limited to one particular GaN LED structure developed by PKU [9], we simulate at least 181 cases based on the micro-structure structures and present very interesting results. In addition, in fabrication etching also involves defects, so the grating structure is not a perfectly periodic crystal. We also develop an error grating model to study the effects of the fabrication randomization. To date, there is no publication on the error grating model of the top and bottom grating structures on GaN LEDs. For all simulations, we calculate the effects on light extraction efficiency with different combinations of grating structures using a finite difference time domain (FDTD) method. In this paper, the simulation model is presented in Section II, top and bottom grating simulation results are presented in Section III, the error grating model is presented in Section IV, and conclusions are presented in Section V.

\section{Simulation MODEL}

\section{A. FDTD}

We analyze a GaN LED device by the Finite Difference Time Domain method. Since FDTD is derived from Maxwell's equations, it can accurately simulate the small grating parameter effects of refraction in device materials, reflection due to linear dispersion or total internal reflection, transmission of escaping light from the LED, and scattering at the grating.

We begin with the differential forms of Maxwell's equations

$$
\begin{aligned}
& \frac{\partial \vec{H}}{\partial t}=-\frac{1}{\mu}(\nabla \times \vec{E}) \\
& \frac{\partial \vec{E}}{\partial t}=\frac{1}{\varepsilon}(\nabla \times \vec{H})-\frac{\sigma}{\varepsilon} \vec{E} .
\end{aligned}
$$

From these equations, we break up the spatial components into each respective axis in the Cartesian coordinate system, which results in the following:

$$
\begin{aligned}
\frac{\partial H_{x}}{\partial t} & =-\frac{1}{\mu}\left(\frac{\partial E_{y}}{\partial z}-\frac{\partial E_{z}}{\partial y}\right) \\
\frac{\partial H_{y}}{\partial t} & =-\frac{1}{\mu}\left(\frac{\partial E_{x}}{\partial z}-\frac{\partial E_{z}}{\partial x}\right) \\
\frac{\partial H_{z}}{\partial t} & =-\frac{1}{\mu}\left(\frac{\partial E_{x}}{\partial y}-\frac{\partial E_{y}}{\partial x}\right) \\
\frac{\partial E_{x}}{\partial t} & =\frac{1}{\varepsilon}\left(\frac{\partial H_{y}}{\partial z}-\frac{\partial H_{z}}{\partial y}-\sigma E_{x}\right) \\
\frac{\partial E_{y}}{\partial t} & =\frac{1}{\varepsilon}\left(\frac{\partial H_{x}}{\partial z}-\frac{\partial H_{z}}{\partial x}-\sigma E_{y}\right) \\
\frac{\partial E_{z}}{\partial t} & =\frac{1}{\varepsilon}\left(\frac{\partial H_{x}}{\partial y}-\frac{\partial H_{y}}{\partial x}-\sigma E_{z}\right) .
\end{aligned}
$$

Next, we employ a Yee's mesh defined as interleaving E and $\mathrm{H}$ component fields [19]. Accuracy of a Yee's mesh is depen-
TABLE I

SIMULATION PARAMETERS FOR BOTH TOP AND BOTTOM GRATINGS

\begin{tabular}{|l|c|c|}
\hline Parameter & Brief Description & Simulated Ranges \\
\hline$A$ & The period of the unit cells & 1 to $6 \mathrm{~mm}$ \\
\hline$w$ & The width of each unit cell & 1 to $6 \mathrm{~mm}$ \\
\hline$d$ & The height of each unit cell & 50 to $200 \mathrm{~nm}$ \\
\hline
\end{tabular}

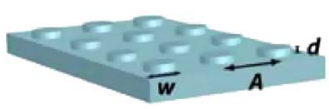

(a)

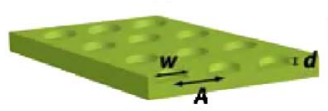

(c)

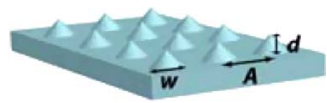

(b)

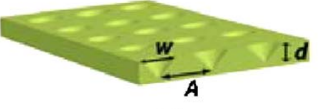

(d)
Fig. 1. Different hole and pillar gratings in our simulation. (a) Cylindrical pillar. (b) Conical pillar. (c) Cylindrical hole. (d) Conical hole.

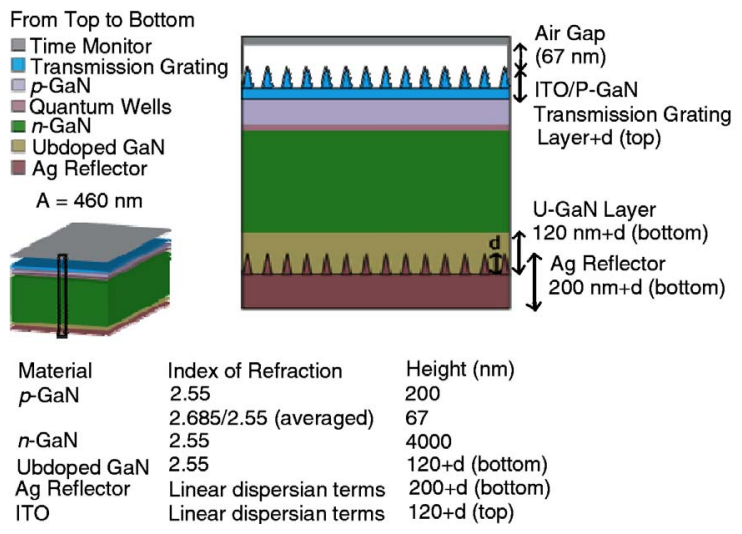

Fig. 2. GaN LED layer model.

dent on the grid size (i.e., on $\Delta x, \Delta y$, and $\Delta z$ ). Based on Yee's implementation, each $\mathrm{H}$ component field is offset by $n+1 / 2$ while the E component fields are offset by $n+1$. The FDTD in conjunction with a Yee's mesh can simulate structures of arbitrary length and size. However, the model is limited in size due to the simulation time and amount of memory required to simulate larger devices. Methods to ease the requirements of 3-D models include use of Graphics Processing Units (GPU), parallel processing, and mode simplifications [21]-[23]. Since 3-D model parameters scale in simulation time like $\mathrm{N}^{4}$ and in memory like $\mathrm{N}^{3}$, the 2-D FDTD is used to simulate this model to avoid unnecessary complications [24].

To define a regular spacing between unit cells in a crystal lattice arrangement, we employ three parameters: unit-cell period $(A)$, unit-cell height $(d)$, and unit-cell width $(w)$, which are shown in Table I and Fig. 1. The unit-cell period, $A$, is the length from center-to-center between unit cells. In 3-D, the parameter $\mathrm{w}$ represents a diameter in the case of a circular structure (i.e., sphere, cone, and cylinder) or a length of a side in the case of a box structure (i.e., cube, rectangular cube). The unit-cell height, $d$, can represent either the depth of the bottom hole or the height of the top pillar. 


\section{B. LED Structure}

The structure of the LED is shown layer-by-layer in Fig. 2, which is based on the Refs. [16], [18] experiment. From bottom to top, the silicon submount is mounted to a $200 \mathrm{~nm}$ layer of silver $(\mathrm{Ag})$ forming both a reflector plate and also filling in holes with a bottom grating height of $d_{\text {bottom }}$ in the $120+d_{\text {bottom }} \mathrm{nm}$ undoped GaN layer (u-GaN), which forms the hole reflection grating structure. Next, a $4 \mu \mathrm{m}$ layer of $n$ type $\mathrm{GaN}(\mathrm{n}-\mathrm{GaN})$ is placed on the hole array, followed by a layer of quantum wells of $67 \mathrm{~nm}$ thickness, and a $200 \mathrm{~nm}$ ptype $\mathrm{GaN}$ layer. The transmission grating structure, of height $d_{\text {top }}$, is composed of a grating structure and mounting material. If ITO is used for the transmission grating, a $200+d_{\text {top }} n$ m layer of ITO is deposited on top of the p-GaN layer, the transmission grating being formed from the ITO layer. If $\mathrm{p}-\mathrm{GaN}$ is used for the transmission grating, the grating can be formed directly on top of the $\mathrm{p}$-GaN layer, making the height of the $\mathrm{p}-\mathrm{GaN}$ layer a total of $200+d_{\text {top }} \mathrm{nm}$. The double grating structures, one on the top and one on the bottom, constitute a so-called transmission-reflection grating or top-bottom grating.

\section{TOP AND BotTOM GRATING Simulation RESUlts}

The simulation starts when the LED turns on from an off state. There is a monitor at a distance of $67 \mathrm{~nm}$, or one grid size distance, above the LED to collect the light emission. For each structure simulation, we must wait until the LED/monitor reaches a steady state, after which we can extract the constant wave $(\mathrm{CW})$ average power. Each simulation sweep of the parameters produces a set of 20,000 data points $(2000 \mathrm{fs}$ at $0.1 \mathrm{fs} / \mathrm{step}$ ), and from this data set, we determine average power over the ending cycle. In this range, maximum steadystate power is radiated due to the $\mathrm{CW}$ source and reflections from the grating. This procedure is done for each case.

There are a total of six transmission gratings and two reflection gratings studied. The transmission gratings are a non-lossy conical P-GaN grating, a non-lossy cylindrical PGaN grating, a non-lossy cylindrical ITO grating, a lossy cylindrical ITO grating, a non-lossy conical ITO grating, and a lossy conical ITO grating. And the reflection gratings are conical reflection grating and cylindrical reflection grating. For each single grating case, we sweep $A$ from 1 to $6 \mu \mathrm{m}, w$ from 1 to $6 \mu \mathrm{m}$ (only for cases where $w<A$ ), and $d=50 \mathrm{~nm}$ to $200 \mathrm{~nm}$. And we give out the optimized structure and list the results in the Table II. The best cases for single gratings from each grating type were taken from our previous studies [16], [25], which are also compared to and agreed with experimental data provided by PKU [9], [18]. References [9] and [18] are the published experimental data on our simulated LED structure. Reference [9] only has electroluminescence data. Reference [18] presents the experimental data of the M-LLO LEDs for the light extraction improvement. The experiment shows about $120 \%$ improvement for a 120-nm-depth grating and $66 \%$ for a $75-\mathrm{nm}$-depth grating [18], which agrees with our simulation. Our simulation gives the following: the light extraction improvement is about $150 \%$ for the 120 -nm-depth grating and $65 \%$ for the $75-\mathrm{nm}$-depth grating [16]. A more detailed comparison of experimental data and simulation are
TABLE II

BEST CASES FOR TOP TRANSMISSION AND BOTTOM REFLECTION GRATINGS

\begin{tabular}{|c|c|c|c|c|c|c|}
\hline & Grating Type & $\begin{array}{c}A \\
\mu \mathrm{m}\end{array}$ & $\begin{array}{c}W \\
\mu \mathrm{m}\end{array}$ & $\begin{array}{c}D \\
\mathrm{~nm}\end{array}$ & $\begin{array}{l}\text { Power } \\
\text { a.u. }\end{array}$ & $\begin{array}{c}\text { Inc. } \\
\%\end{array}$ \\
\hline \multirow[t]{6}{*}{$\begin{array}{l}\text { Transmission } \\
\text { (top) }\end{array}$} & $\begin{array}{c}\text { Non-Lossy } \\
\text { Conical p-GaN }\end{array}$ & 1 & 1 & 138 & 12.374 & 165 \\
\hline & $\begin{array}{c}\text { Non-Lossy } \\
\text { Cylindrical } \\
\text { p-GaN }\end{array}$ & 2 & 1 & 156 & 10.926 & 134 \\
\hline & $\begin{array}{l}\text { Non-Lossy } \\
\text { Cylindrical } \\
\text { ITO }\end{array}$ & 1 & 1 & 126 & 9.7258 & 109 \\
\hline & $\begin{array}{c}\text { Lossy } \\
\text { Cylindrical } \\
\text { ITO }\end{array}$ & 1 & 1 & 118 & 9.0752 & 95 \\
\hline & $\begin{array}{c}\text { Non-Lossy } \\
\text { Conical ITO }\end{array}$ & 4 & 4 & 166 & 8.8755 & 90 \\
\hline & $\begin{array}{c}\text { Lossy Conical } \\
\text { ITO }\end{array}$ & 4 & 4 & 164 & 8.4015 & 80 \\
\hline \multirow[t]{2}{*}{$\begin{array}{l}\text { Reflection } \\
\text { (bottom) }\end{array}$} & Conical & 1 & 1 & 200 & 10.13 & 117 \\
\hline & Cylindrical & 2 & 1 & 136 & 9.8135 & 111 \\
\hline Reference & None & - & - & - & 4.6608 & 0 \\
\hline
\end{tabular}

presented in [16]. Our simulation also shows that the single grating can achieve about $80-165 \%$ light extraction efficiency improvement. Based on the above information, the best-case top transmission grating was paired with a best-case bottom reflection grating to form a transmission-reflection grating, a total of 12 transmission-reflection grating combinations. In the simulation, lossy materials have a loss factor of $|\alpha| \neq 0$, while the non-lossy materials have $\alpha=0$. The extinction coefficient, or the imaginary portion of the index of refraction $n$, is defined as $\alpha: n_{\text {comp }}=n_{\text {real }}+\mathrm{j}^{*} \alpha$. In our simulation, the $\alpha$ factor of lossy ITO is $0.01 \mathrm{~dB} / \mathrm{mm}$. "Conical" and "Cylindrical" refer to grating cell shapes. Transmission grating shapes refer to "pillars," while reflection grating shapes refer to "holes." Reflection grating materials are always u-GaN holes mounted and filled in with Ag. The p-GaN transmission grating is formed from the p-type GaN semiconductor, while the ITO transmission grating is formed from a deposited layer of ITO on top of the p-GaN layer. The optimized single grating suggested from our simulation is the $\mathrm{p}-\mathrm{GaN}$ conical top grating; the next one is the top cylindrical p-GaN. The conical top ITO gratings have less light improvement compared to all the other top and bottom single grating cases. The bottom reflection gratings also give reasonable good results on light extraction improvement, which is about $117 \%$. In general, we found $A=1 \mu \mathrm{m}$ gives the best results for single grating simulations as shown in Table II, which suggest a nanostructure grating may be the future trend for better light extraction improvement.

We simulate 181 cases in total with different $\mathrm{A}$, w, d values, including the single top, the single bottom, and the top-bottom gratings. Then, we rank all of them based on light extraction improvement. The brief results of the doublegrating simulation are shown in Table III. The best-case top transmission gratings are matched with the best-case bottom 
TABLE III

TOP/BOTTOM GRATING OPTIMIZATION RESULTS

\begin{tabular}{|l|c|c|c|c|c|c|c|c|c|c|}
\hline $\begin{array}{l}\text { Case } \\
\text { No. }\end{array}$ & $\begin{array}{c}\text { Structure Name } \\
\text { Top Transmission }\end{array}$ & $\begin{array}{c}A \\
\mu \mathrm{m}\end{array}$ & $\begin{array}{c}w \\
\mu \mathrm{m}\end{array}$ & $\begin{array}{c}d \\
\mathrm{~nm}\end{array}$ & $\begin{array}{c}\text { Structure Name } \\
\text { Bottom Reflection }\end{array}$ & $\begin{array}{c}A \\
\mu \mathrm{m}\end{array}$ & $\begin{array}{c}w \\
\mu \mathrm{m}\end{array}$ & $\begin{array}{c}d \\
\mathrm{~nm}\end{array}$ & $\begin{array}{c}\text { Power } \\
\text { a.u. }\end{array}$ & $\begin{array}{c}\text { Inc. } \\
\%\end{array}$ \\
\hline 1 & Lossy Conical ITO & 4 & 4 & 164 & Cylindrical & 2 & 1 & 136 & 6.6252 & 42 \\
\hline 2 & Non-Lossy Conical P-GaN & 1 & 1 & 138 & Cylindrical & 2 & 1 & 136 & 6.2106 & 33 \\
\hline 3 & Non-Lossy Conical ITO & 4 & 4 & 166 & Cylindrical & 2 & 1 & 136 & 6.1401 & 32 \\
\hline 4 & Non-Lossy Cylindrical P-GaN & 2 & 1 & 156 & Cylindrical & 2 & 1 & 136 & 6.1368 & 32 \\
\hline 5 & Non-Lossy Cylindrical ITO & 1 & 1 & 126 & Cylindrical & 2 & 1 & 136 & 5.5491 & 19 \\
\hline 6 & Lossy Cylindrical ITO & 1 & 1 & 118 & Cylindrical & 2 & 1 & 136 & 5.5181 & 18 \\
\hline 7 & Non-Lossy Conical ITO & 4 & 4 & 166 & Conical & 1 & 1 & 200 & 5.4614 & 17 \\
\hline 8 & Lossy Conical ITO & 4 & 4 & 164 & Conical & 1 & 1 & 200 & 5.3947 & 16 \\
\hline 9 & Non-Lossy Conical P-GaN & 1 & 1 & 138 & Conical & 1 & 1 & 200 & 5.2641 & 13 \\
\hline 10 & Non-Lossy Cylindrical P-GaN & 2 & 1 & 156 & Conical & 1 & 1 & 200 & 5.2185 & 12 \\
\hline 11 & Non-Lossy Cylindrical ITO & 1 & 1 & 126 & Conical & 1 & 1 & 200 & 5.0291 & 8 \\
\hline 12 & Lossy Cylindrical ITO & 1 & 1 & 118 & Conical & 1 & 1 & 200 & 4.8967 & 5 \\
\hline 13 & Non-grating (Reference) & - & - & - & None & - & - & - & 4.6608 & 0 \\
\hline 14 & Lossy Cylindrical ITO & 1 & 1 & 186 & Conical & 1 & 1 & 200 & 4.2404 & -9 \\
\hline
\end{tabular}

reflection gratings to show effects of both together, and results are reported in percent increase with respect to the nongrating case. There are 12 combinations of six transmission gratings and two reflection gratings studied, which are listed as Cases 1-12 in Table III. These types of gratings show significant improvement over the non-grating case, or case 13 in Table III. The structure types by ranking show that cylindrical reflection gratings are preferred when coupled with a transmission grating structure since ranks 1 to 6 all have a cylindrical reflection grating. Although conical reflection gratings sometimes perform better as a single grating case, this simulation reveals that optimal conditions for a single grating do not always apply for the transmission-reflection grating case. Also, conical transmission gratings perform better than cylindrical transmission gratings when combined with bottom reflection gratings. The top three ranks are dominated by conical structures. And the following three rankings are all cylindrical transmission gratings.

Since both the top and the bottom grating change the optical field distribution in the non-grating LED and influence the light extraction, they have coupling effects and need to be matched in design. Otherwise, they may not improve light extraction. In general, there is a clear trend that shows conical transmission gratings and cylindrical reflection gratings are preferred. However, the results of the transmissionreflection gratings, derived from the best cases of reflection and transmission gratings only, perform more poorly (about $42 \%$ maximum) than just the single grating structures alone. This can be explained by the trapped optical field changing with grating structure, since the scattering due to the grating is dependent on the grating structure parameters. The optimal conditions that produce the greatest light emission in a single grating case do not necessarily apply to the transmissionreflection grating case. When a reflection grating is paired with a transmission grating, the optical fields are altered within the device, therefore producing unfavorable light extraction due to the poor coupling or the mismatch between the gratings and the newly reorganized optical fields. In the worst case, a destructive interference or mismatch will degrade light extraction efficiency, which is also presented in Table III (Case
14), for example. This last ranked structure even has worse light extraction efficiency (about -9\%) than the non-grating case.

The best performing grating of this transmission-reflection simulation set uses lossy conical-shaped ITO pillars as a transmission grating material matched with cylindrical-shaped $\mathrm{u}-\mathrm{GaN}$ holes as a reflection grating. This type of grating has a $42 \%$ improvement over the non-grating case. This is an interesting result, since the both lossy and non-lossy conical ITO transmission gratings ranked lowest in the transmission grating. The difference between the lossy and non-lossy materials is an imaginary loss factor in the index of refraction described by

$$
n_{\text {comp }}=n_{\text {real }}+j n_{\text {imag }}
$$

where $n_{\text {imag }}=\frac{\gamma \lambda}{4 \pi}$, and $\gamma$ is the exponential loss factor in units of $\mu \mathrm{m}^{-1}$ and $\lambda$ is the wavelength of light. It is possible that evanescent waves are penetrating the thin grating structure, causing greater light extraction efficiency. Also, the optical field for the cylindrical reflection grating may match better to the lossy transmission grating than the non-lossy transmission grating. In addition, the worse single grating combination may have better potential to improve its light extraction efficiency compared the best single grating case.In the total rankings of all simulated cases, the best single transmission and the best single reflection grating combination ranks only at 158 out of 181 total cases simulated, which is rank 9 in Table III. Clearly, the direct addition of optimal grating structures does not linearly add to the total output power. The optical fields have an optimal set of grating parameters that may be disturbed when another grating is introduced. It is possible that another set of optimal grating conditions exist, but it must be discovered by a thorough sweep of all top and bottom grating parameters (six parameters in total). This is very important and critical in the double-grating LED design. But it would take an extraordinary amount of time. It is clear now that the transmission-reflection gratings simulated here are not overall-optimal grating structures, since none of them outperform the single grating cases. 


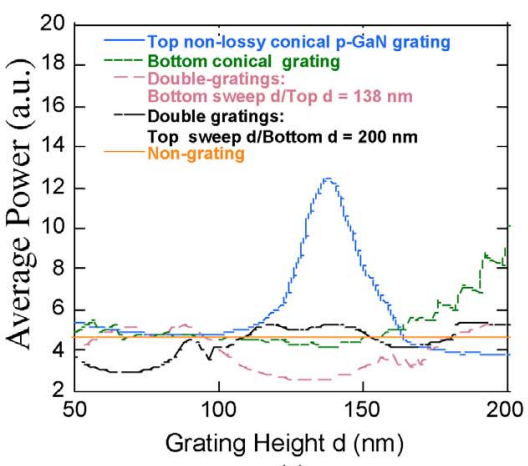

(a)

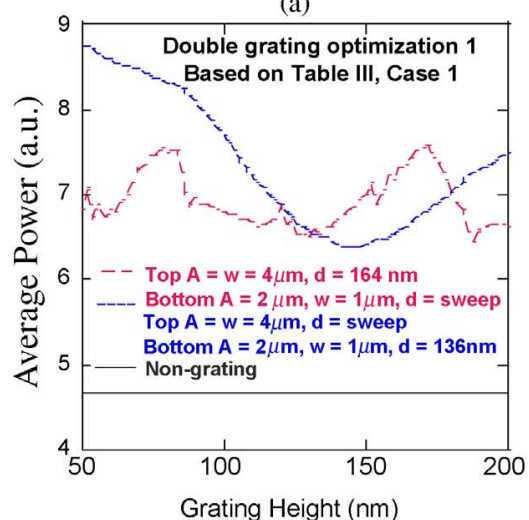

(b)

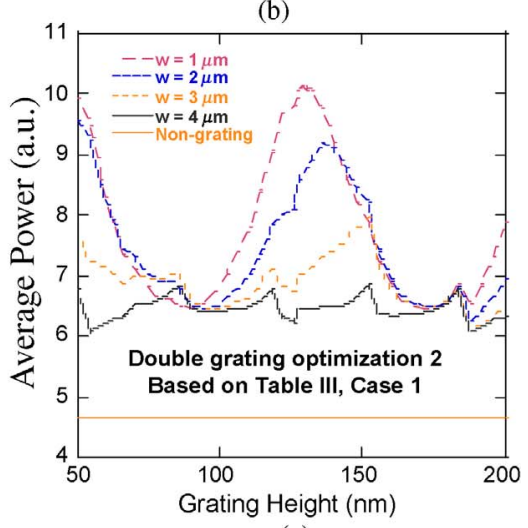

(c)

Fig. 3. (a) Comparison gratings with only top, only bottom, and double gratings based on Table III (Case 9) (both top and bottom grating $A=1 \mu \mathrm{m}$, $w=1 \mu \mathrm{m}$ ). (b) Double grating optimization 1 based on Table III (Case 1), sweeping either bottom grating $\mathrm{d}$ or top grating $\mathrm{d}$. (c) Double grating optimization 2 based on Table III (Case 1), top grating $A=w=4 \mu \mathrm{m}$, $d=52 \mathrm{~nm}$ and bottom grating $A=4 \mu \mathrm{m}, w=$ sweep, $d=$ sweep.

Fig. 3(a) shows an example of above case and explains the concept of mismatching. Here, we sweep the top grating height with the bottom grating height fixed and vice versa. The best top grating, non-lossy conical $\mathrm{p}-\mathrm{GaN}$ transmission grating, has optimal parameters of $A=1 \mu \mathrm{m}, w=1 \mu \mathrm{m}$, $d=138 \mathrm{~nm}$. If we vary $d$ as a fabrication error from $118 \mathrm{~nm}$ to $186 \mathrm{~nm}$, then even slight variations result in, not only a non-optimal grating, but also the worst case. The best bottom grating, conical reflection grating, has best light extraction at $A=1 \mu \mathrm{m}, w=1 \mu \mathrm{m}, d=200 \mathrm{~nm}$ (a limit of our simulation). These two gratings are totally mis-matched. Therefore, when combining them, we only can achieve 13\% (5.26 a.u. power output, Case 9 in Table III) of light extraction improvement, which is much lower than that of the optimized single gratings. Fig. 3(a) also shows that the nano-level variation of depth can be a primarily influence of mismatching degree between optical fields. The experimental data published in [18] also supports the strong dependence of light extraction efficiency on the grating depth. We also perform some double grating optimization simulations based on Case 1 in Table III by varying one parameter at a time. We do not cover all the parameter's possible values, but we can still show the optimized double grating can be better than the single gratings. The results are shown in Fig. 3(b) and (c). With the optimization of only the height value, we can achieve 8.76 a.u. power output, which is an $87 \%$ improvement. In addition, we also sweep $\mathrm{A}$ and $\mathrm{w}$ of bottom grating with fixed top grating and fixed $\mathrm{d}$ of bottom grating. When the top lossy conical ITO grating at $A=w=4 \mu \mathrm{m}, d=52 \mathrm{~nm}$ and bottom cylindrical reflection grating at $A=4 \mu \mathrm{m}, w=1 \mu \mathrm{m}, d=136 \mathrm{~nm}$, the power output is 9.76 a.u., which is about a $110 \%$ improvement. Finally, a further optimization with the same transmission grating but varying cylindrical reflection grating shows that $A=4, w=1, d=128 \mathrm{~nm}$ gives an improvement of $118 \%$, as shown in Fig. 3(c). This result is not an absolute maximum which should be found from a thorough sweep of all top and bottom grating parameters' possible values. However it is still better than the optimized single grating case, which is $80 \%$ for this type of single top grating and $111 \%$ for this type of single bottom grating. All these calculations show that the optimization of a double grating is much more sensitive and crucial than the best single grating pair and can give better results.

To further prove field variation theory, we output the optical field distribution of GaN LEDs. First, the optical field distributions in the top grating only in Fig. 4(a) and bottom grating only in Fig. 4(b) are very different from each other. When the top grating and bottom grating are added together as in Table III (Case 1), shown in Fig. 4(c), the optical field distribution will not prefer either top grating or bottom grating. An optical field cancelation effect (rather than enhancement effect) may give a light extraction efficiency of $42 \%$, which is much lower than single grating cases. Second, the best top or bottom-grating optical field distributions are shown in Fig. 4(d) or (e). Compared to Fig. 4(a) or (b), optical field of (d) or (e) is less confined in the active region. There is more optical field distribution variation between (a) and (d), compared to that of (b) and (e). There is also more light extraction improvement difference between (a) at $80 \%$ and (d) at $165 \%$, compared to that of (b) at $111 \%$ and (e) at $117 \%$, as well. Finally, we present the non-grating LED case in Fig. 4(f), which has the strongest field around the active region compared to all other cases. In general, our simulation shows that better light extraction has more optical fields outside the active region. The top grating optical field and bottom grating optical field in our simulation may not matched, which are very interesting results. The exact correlation of the double grating design, optical field, and light extraction efficiency requires intensive simulation, which is outside the scope of this paper.

It is very surprising that the best single grating is not related to the best double grating at first. This result is very 


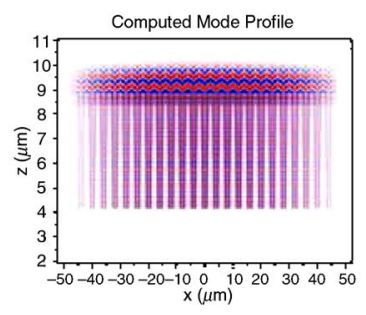

(a)

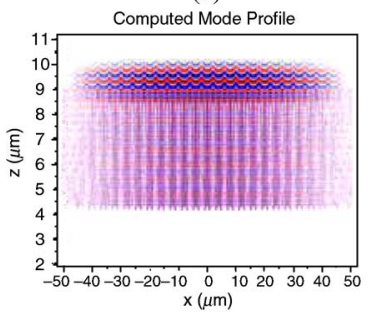

(c)

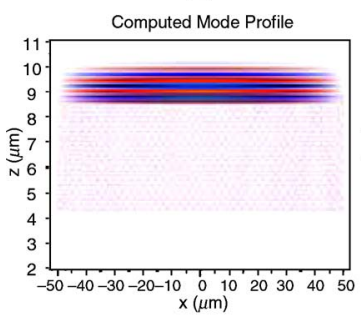

(e)

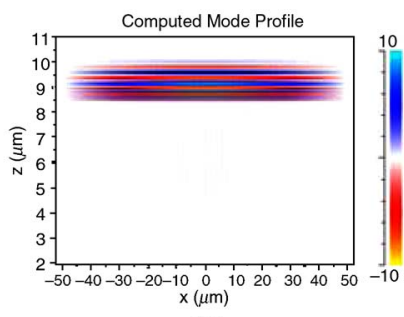

(b)

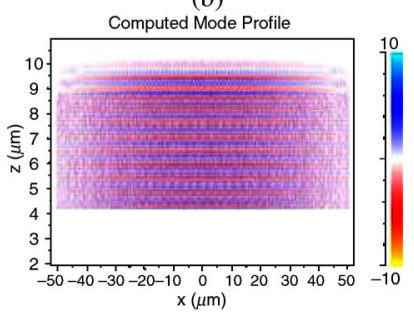

(d)

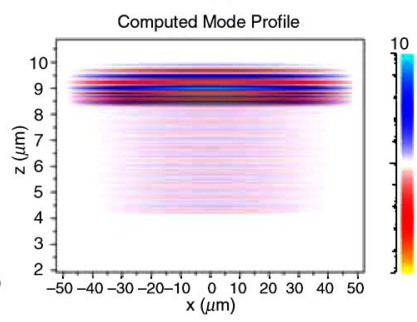

(f)
Fig. 4. (a) Top conical grating only $A=4 \mu \mathrm{m}, w=4 \mu \mathrm{m}, d=164 \mathrm{~nm}$. (b) Bottom cylindrical grating only $A=2 \mu \mathrm{m}, w=1 \mu \mathrm{m}, d=136 \mathrm{~nm}$. (c) Top and bottom grating (a) $+(\mathrm{b})$, Table III (Case 1). (d) Best top grating: conical $A=1 \mu \mathrm{m}, w=1 \mu \mathrm{m}, d=138 \mathrm{~nm}$ (Table II). (e) Best bottom grating: conical $A=1 \mu \mathrm{m}, w=1 \mu \mathrm{m}, d=200 \mathrm{~nm}$ (Table II). (f) Non-grating LED case.

useful for most people in the field, since there are now many experimental results on single gratings. It is very easy to design a double grating using the best single gratings, which can complicate the fabrication procedure. However, there is not much gain in overall performance of LEDs, since the effects of grating structures are not necessarily linearly additive to improve light extraction efficiency. Before fabrication, detailed studies need to be carried out to determine maximum coupling efficiency according to the optical field variation when adding an additional grating structure. Also, matching grating is a very important concept in double-grating design. As long as you can match them, it is not critical which grating you choose according to your fabrication capability. Therefore, the grating design is very open in this sense.

\section{EFFECTS OF RANDOMIZATION ON TOP AND BOTTOM GRATINGS}

To further understand the effects of fabrication defects on transmission-reflection gratings, we simulate the transmissionreflection grating model with each cell randomly shifting a distance along the axis in varying degrees of randomization intensity to create an error grating model. While the widths of holes can be fabricated to great precision, often the placement of holes is a cause for concern, as it affects light extraction efficiency of otherwise ordered photonic crystal structures. The

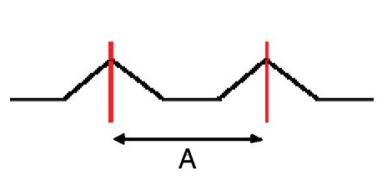

(a)

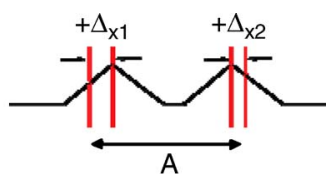

(b)

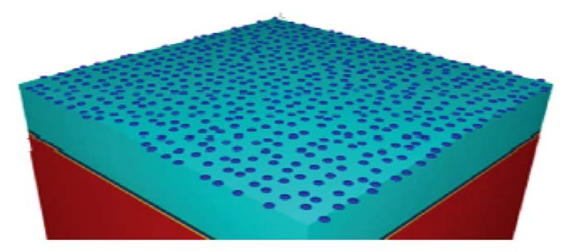

(c)

Fig. 5. Error grating model. (a) Normal reference grating model. (b) Error grating model with both positive and negative shifts. (c) Error grating model on GaN LED.

error grating model is very important to explain the difference between model/optimization and fabrication.

Our simulation is still based on the 2-D FDTD method. An example of random displacements is shown in Fig. 5(b) with a normal grating as a reference in Fig. 5(a). Displacements can move either way from the original center point. The error grating model shows examples of a positive and negative $\Delta_{\mathrm{X}}$ shift. This randomization is applied to all grating cells in the photonic crystal arrangement with the following equation:

$$
x_{\mathrm{pos}}=N^{*} \text { period }+\left(2^{*} \text { rand }-1\right)^{*} R^{*} \text { period }
$$

where $N$ is an integer index defining the original grating cell location, period is the grating period $(A)$, rand is a pseudo-randomly generated number from 0 to 1 , and $R$ is the randomization factor from 0 to 1 . The quantity $\Delta_{\mathrm{X}}$ represents $(2 * \text { rand }-1)^{*} R^{*}$ period in (4). By varying $R$, which applies to all grating cells, from 0 to 1 in 40 steps, the individual rand factor can be emphasized or deemphasized. This process is repeated for each of the 12 transmission-reflection grating models. And above error grating models are placed into $\mathrm{GaN}$ LED device models as shown in Fig. 5(c) to calculate light extraction efficiency.

The effects of randomization in gratings, in general, appear to help light extraction efficiency, peaking at about a randomization factor of $10 \%$ in most simulations. Fig. 6 shows the results for each transmission and reflection grating pair. All data are normalized according to its $R=0$ values for easy reading. Fig. 6(a) shows the results for a conical reflection grating matched with each optimal transmission grating. These top-bottom gratings combinations are Case 12, Case 8, Case 11, Case 7, Case 10, and Case 9 in Table III. Fig. 6(b) shows the results for a cylindrical grating paired with each optimal transmission grating. These are Case 6, Case 1, Case 5, Case 3 , Case 4, and Case 2. As the randomization intensity factor is increased from 0 to 1 , many transmission-reflection gratings experience an increase in light extraction efficiency around a $10 \%$ displacement of grating cells. In essence, a slight random variation or fabrication defect in grating cells would not only be beneficial but desirable for many transmission-reflection grating types up to a variation of $10-15 \%$ for most double 


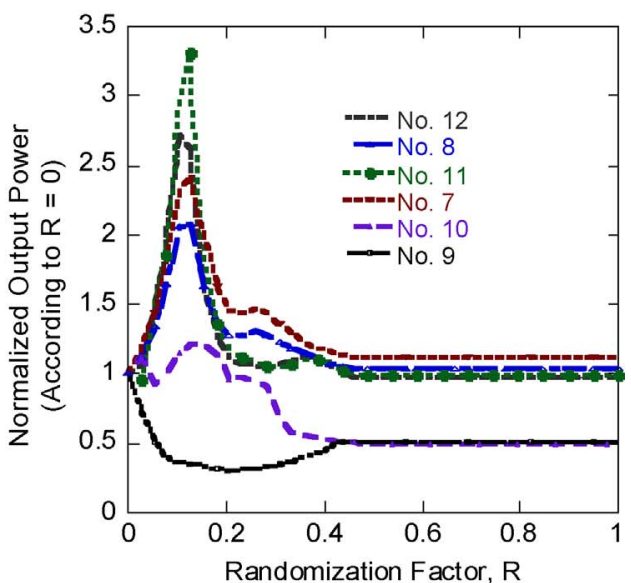

(a)

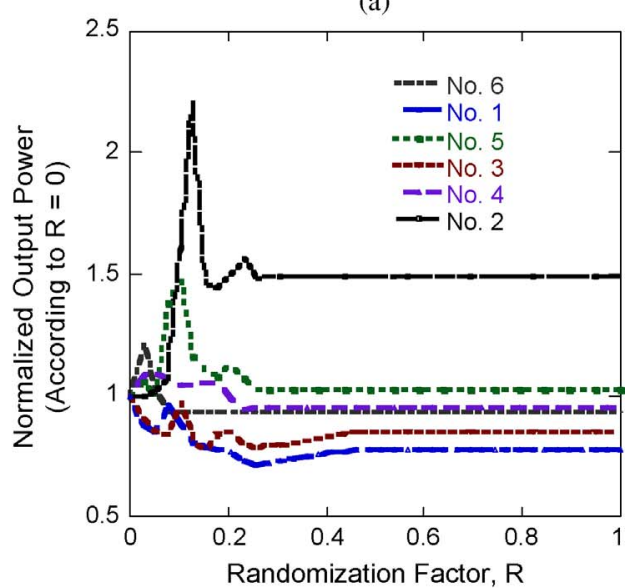

(b)

Fig. 6. Results of error grating simulation for (a) conical reflection gratings paired with transmission gratings: Case 12, Case 8, Case 11, Case 7, Case 10, and Case 9 in Table III. (b) Cylindrical grating paired with transmission grating: Case 6, Case 1, Case 5, Case 3, Case 4, and Case 2 in Table III.

grating cases. The best case is the non-lossy cylindrical ITO transmission and conical reflection gratings which can reach up to a $230 \%$ improvement over the original transmissionreflection grating model at a randomization intensity factor of $12.8 \%$. Also, after $R=10 \%$, light extraction efficiency drops, and after $R=50 \%$ settles to a steady state value. Usually, the randomization creates local variation of grating structure, $A$ and $w$. As top and bottom gratings are only optimized separately, it is possible that a small local perturbation could result in more matching gratings and could introduce local light extraction improvement. A conical single reflection grating has lower performance than that of a cylindrical grating. It has more benefit from a randomization of the grating.

One case that did not improve at around $R=10 \%$ is the case of a non-lossy P-GaN conical top grating $(A=1 \mu \mathrm{m}$, $w=1 \mu \mathrm{m}, d=138 \mathrm{~nm})$ and the conical reflection grating $(A=1 \mu \mathrm{m}, w=1 \mu \mathrm{m}, d=200 \mathrm{~nm})$. In the single grating simulations, the respective reflection and transmission gratings represent the best case, as shown in Table II. Since these gratings are already optimized to the best case in the single grating case, the randomization effect is not strong enough to offset destruction from the double gratings. On the other hand it may further decrease light extraction efficiency by adding more local destruction or mismatching, hence the drop shown in Fig. 6(a) as the randomization intensity factor increased.

Experimentally, the double grating could give a comparably higher output power as compared with the single grating one, when some suitable grating/texturing processes are used on both the top ITO/p-GaN and bottom substrate layers. In this paper, we only prove that the best single grating is not necessarily the best double grating option in Section III. As for the experiment, we show here that if fabrication is not perfect, the light output variation can be about $230 \%$ improvement in our double grating error model. Therefore, the experimental data of a double grating can be better than a single grating in either matching grating concepts, error grating effects, or both.

Randomization actually has a positive effect on many transmission-reflection grating structures for randomization factors of 10-15\%. It increases light extraction efficiency while having the added benefit of alleviating some of the fabrication complexities demanded by strict periodicities in photonic crystal LEDs. Until now, we have not found any papers on the randomization model or our so called error model in GaN LEDs. We believe the LED error grating model is unique. There exists no experimental data which claims error percentage in their fabrication process. Nevertheless, our model can be used to predict the fabrication error or used as data analysis in the real design in the future.

\section{CONCLUSION}

Transmission-reflection grating structures, taken from the optimized cases of transmission and reflection gratings alone, are simulated using FDTD. In general, grating structures will improve light extraction of LEDs compared to non-grating structure. The simulation results also show that the optimized single grating optical fields are altered significantly by the introduction of a second grating structure. The double grating can achieve a light extraction improvement of $118 \%$ with respect to the non-grating case. One of the worst double grating cases is can reduce light extraction efficiency by $9 \%$ respect to the non-grating case. Therefore, all of the grating parameters would need to be swept for both transmission and reflection grating cases simultaneously in terms of A, w, and $\mathrm{d}$ in order to find the optimized double-grating structure.

We also present error grating models. A fabrication defect of around $10 \%$ actually helps increase light extraction efficiency. In the best case, $230 \%$ improvement over the original transmission-reflection grating model can be achieved. Not all models exhibit such an increase. In general, fabrication of photonic crystal LEDs do not necessarily need to keep photonic crystal arrangements so ordered and exact. Low intensity random defects in periodic photonic crystals are not only beneficial but desirable in many grating structures.

\section{ACKNOWLEDGMENT}

The authors would like to thank Prof. B. Zhang, Dr. X. N. Kang, and Dr. G. Y. Zhang from Peking University for 
providing expert advice on the GaN LED grating experiments and modeling.

\section{REFERENCES}

[1] O. Svensk, P. T. Torma, S. Suihkonen, M. Ali, H. Lipsanen, M. Sopanen, A. Odnoblyudov, and V. E. Bougrov, "Enhanced electroluminescence in $405 \mathrm{~nm} \mathrm{InGaN/GaN} \mathrm{LEDs} \mathrm{by} \mathrm{optimized} \mathrm{electron} \mathrm{blocking} \mathrm{layer,"} J$. Crystal Growth, vol. 310, no. 23, pp. 5154-5157, 2008.

[2] R. H. Horng, C. C. Yang, J. Y. Wu, S. H. Huang, C. E. Lee, and D. S. Wuu, "GaN-based light-emitting diodes with indium tin oxide texturing window layers using natural lithography," Appl. Phys. Lett., vol. 86, no. 22, p. 221101, 2005.

[3] H. W. Huang, C. C. Kao, J. T. Chu, H. C. Kuo, S. C. Wang, and C. C. $\mathrm{Yu}$, "Improved light-output and electrical performance of InGaN-based light-emitting diode by microroughening of the p-GaN surface," IEEE Photon. Technol. Lett., vol. 17, no. 5, pp. 9383-9385, May 2005.

[4] T. X. Lee, C. Y. Lin, S. H. Ma, and C. C. Sun, "Analysis of positiondependent light extraction of GaN-based LEDs," Opt. Express, vol. 13, no. 11, pp. 4175-4179, May 2005.

[5] G. Hatakoshi, Y. Hattori, S. Saito, N. Shida, and S. Ninoue, "Device simulator for designing high-efficiency light-emitting diodes," Jpn. J. Appl. Phys., vol. 46, no. 8B, pp. 5419-5425, 2007.

[6] Y. A. Chen, N. Y. Liang, L. H. Laih, W. C. Tsay, M. N. Chang, and J. W. Hong, "Improvement of electroluminescence characteristics of porous silicon LED by using amorphous silicon layers," Elec. Lett., vol. 33, no. 17, pp. 1489-1490, 1997.

[7] Y. A. Chen, N. Y. Liang, L. H. Laih, W. C. Tsay, M. N. Chang, and J. W. Hong, "Improvement of current injection of porous silicon," Jpn. J. Appl. Phys., vol. 36, pp. 1574-1577, Jan. 1997.

[8] H. W. Huang, C. C. Kao, H. C. Chu, S. C. Wang, and C. C. $\mathrm{Yu}$, "Improvement of InGaN-GaN light-emitting diode performance with a nano-roughened p-GaN Surface," IEEE Photon. Technol. Lett., vol. 17, no. 5, pp. 983-985, May 2005.

[9] K. Bao, X. N. Kang, B. Zhang, T. Dai, C. Xiong, H. Ji, G. Y. Zhang, and Y. Chen, "Improvement of light extraction from patterned polymer encapsulated GaN-based flip-chip light-emitting diodes by imprinting," IEEE Photon. Technol. Lett., vol. 19, no. 22, pp. 1840-1842, Nov. 2007.

[10] H. Y. Ryu, J. K. Kwang, Y. J. Lee, and Y. H. Lee, "Enhancement of light extraction from 2-D photonic crystal slab structures," IEEE J. Sel. Top. Quantum Electron., vol. 8, no. 2, pp. 231-237, Mar.-Apr. 2002.

[11] T. Kim, A. J. Danner, and K. D. Choquette, "Enhancement in external quantum efficiency of blue light-emitting diode by photonic crystal surface grating," Electron. Lett., vol. 41, no. 20, pp. 1138-1139, 2005.

[12] F. F. Ren, M. B. Yu, J. D. Ye, Q. Chen, G. Q. Lo, and D. L. Kwong, "Enhanced vertical light extraction from ultrathin amorphous $\mathrm{Si}-\mathrm{Si}_{3} \mathrm{~N}_{4}$ multilayers with photonic crystal patterns," IEEE Photon. Technol. Lett., vol. 21, no. 2, pp. 91-93, Jan. 2009.

[13] T. Dai, B. Zhang, Z.-S. Zhang, D. Liu, X. Wang, K. Bao, X.-N. Kang, J. $\mathrm{Xu}$, D.-P. Yu, and X. Zhu, "Surface light extraction mapping from 2-D array of 12 -fold photonic quasicrystal on current injected GaN-based LEDs," Chin. Phys. Lett., vol. 24, no. 4, pp. 979-982, 2007.

[14] R. J. Yan and Q. K. Wang, "Enhancement of light extraction efficiency in OLED with periodic nano-structure," in Proc. IEEE Int. Conf. Nano/Micro Eng. Molecular Syst., Jan. 2006, pp. 713-716.

[15] J. Q. Xi, H. Luo, A. J. Pasquale, J. K. Kim, and E. F. Schubert, "Enhanced light extraction in GaInN light-emitting diode with pyramid reflector," IEEE Photon. Technol. Lett., vol. 18, no. 22, pp. 2347-2349, Nov. 2006.
[16] S. Trieu, X. M. Jin, B. Zhang, T. Dai, K. Bao, X. N. Kang, and G. Y. Zhang, "Light extraction improvement of GaN-based light emitting diodes using patterned undoped GaN bottom reflection gratings," Proc. SPIE, vol. 7216, no. 2Q, pp. 1-8, 2009.

[17] D. A. Steigerwald, J. C. Bhat, D. Collins, R. M. Fletcher, M. O. Holcomb, M. J. Ludowise, P. S. Martin, and S. L. Rudaz, "Illumination with solid state lighting technology," IEEE J. Sel. Top. Quantum Electron., vol. 8, no. 2, pp. 310-320, Mar-Apr. 2002.

[18] K. Bao, X. Kang, B. Zhang, T. Dai, Y. Sun, Q. Fu, G. Lian, G. Xiong, G. Zhang, and Y. Chen, "Improvement of light extraction from GaNbased thin-film light-emitting diodes by patterning undoped $\mathrm{GaN}$ using modified laser lift-off," Appl. Phys. Lett., vol. 92, no. 14, p. 141104, 2008.

[19] K. Yee, "Numerical solution of initial boundary value problems involving Maxwell's equations in isotropic media," IEEE Trans. Antennas Propag., vol. 14, no. 3, pp. 302-307, May 1966.

[20] S. Kim, K. Lee, J. Kim, M. Kwon, and S. Park, "Fabrication of photonic crystal structures on light emitting diodes by nanoimprint lithography," Nanotechnology, vol. 18, no. 5, p. 055306, 2007.

[21] S. Adams, J. Payne, and R. Boppana, "Finite difference time domain (FDTD) simulations using graphics processors," in Proc. HPCMP Users Group Conf., 2007, pp. 334-338.

[22] Z. M. Liu, A. S. Mohan, T. A. Aubrey, and W. R. Belcher, "Techniques for implementation of the FDTD method on a CM-5 parallel computer," IEEE Ant. Prop. Mag., vol. 37, no. 5, pp. 64-71, Oct. 1995.

[23] Y. Chen, T. L. Simpson, and T. Q. Ho, "Highly efficient technique for solving radiation and scattering problems," IEEE Proc., vol. 139, no. 1, pp. 7-10, Feb. 1992.

[24] H. Kimura and N. Yoshida, "3-D full-wave analysis with nonlinearity and line characteristics of device by electromagnetic field analysis on time domain," Elec. Comm. Jpn., vol. 75, pp. 89-100, Mar. 1991.

[25] X. Jin, B. Zhang, T. Dai, W. Wei, X. N. Kang, G. Y. Zhang, S. Trieu, and F. Wang, "Optimization of top polymer gratings to improve $\mathrm{GaN}$ LEDs light transmission," Chin. Opt. Lett., vol. 6, no. 10, pp. 788-790, 2008. 